\title{
Reproducibility and Reuse of Adaptive Immune Receptor Repertoire Data
}

Felix Breden ${ }^{1 *}$, Eline T. Luning Prak ${ }^{2 *}$, Bjoern Peters ${ }^{3}$, Florian Rubelt ${ }^{4}$, Chaim A. Schramm ${ }^{5}$, Christian E. Busse ${ }^{6}$, Jason A. Vander Heiden ${ }^{7}$, Scott Christley ${ }^{8}$, Syed Ahmad Chan Bukhari ${ }^{9}$, Adrian Thorogood ${ }^{10}$, Frederick A. Matsen IV ${ }^{11}$, Yariv Wine ${ }^{12}$, Uri Laserson ${ }^{13}$, David Klatzmann ${ }^{14}$, Daniel C. Douek ${ }^{5}$, Marie-Paule Lefranc ${ }^{15}$, Andrew M. Collins ${ }^{16}$, Tania Bubela ${ }^{17}$, Steven H. Kleinstein ${ }^{9}$, Corey T. Watson ${ }^{18}$, Lindsay G. Cowell ${ }^{8}$, Jamie K. Scott ${ }^{19}$ and Thomas B. Kepler ${ }^{20,21}$

${ }^{1}$ Department of Biological Sciences, Simon Fraser University, Burnaby, BC, Canada, ${ }^{2}$ Department of Pathology and Laboratory Medicine, Perelman School of Medicine, University of Pennsylvania, Philadelphia, PA, United States, ${ }^{3}$ La Jolla Institute for Allergy and Immunology, La Jolla, CA, United States, ${ }^{4}$ Department of Microbiology and Immunology, Institute for Immunity, Transplantation and Infection, Stanford University School of Medicine, Stanford, CA, United States, ${ }^{5}$ Vaccine Research Center, National Institute of Allergy and Infectious Diseases (NIAID), National Institutes of Health (NIH), Bethesda, MD, United States, ${ }^{6}$ Division of B Cell Immunology, Deutsches Krebsforschungszentrum (DKFZ), Heidelberg, Germany, ${ }^{7}$ Department of Neurology, Yale University School of Medicine, New Haven, CT, United States, ${ }^{8}$ Department of Clinical Sciences, University of Texas Southwestern Medical Center, Dallas, TX. United States, ${ }^{9}$ Department of Pathology, Yale University School of Medicine, New Haven, CT, United States, ${ }^{10}$ Centre of Genomics and Policy, McGill University, Montreal, QC, Canada, " Public Health Sciences Division and Computational Biology Program, Fred Hutchinson Cancer Research Center, Seattle, WA, United States, ${ }^{12}$ Department of Molecular Microbiology and Biotechnology, Tel Aviv University, Tel Aviv, Israel, ${ }^{13}$ Department of Genetics and Genome Sciences, Icahn School of Medicine at Mount Sinai, New York, NY, United States, ${ }^{14}$ Immunology-Immunopathology-Immunotherapy (i3 \& i2B), Sorbonne Université, Paris, France, ${ }^{15}$ IMGT, LIGM, Institut de Génétique Humaine IGH, CNRS, University of Montpellier, Montpellier, France, ${ }^{16}$ School of Biotechnology and Biomolecular Sciences, University of New South Wales, Kensington, NSW, Australia, ${ }^{17}$ Faculty of Health Sciences, Simon Fraser University, Burnaby, BC, Canada, ${ }^{18}$ Department of Biochemistry and Molecular Genetics, University of Louisville School of Medicine, Louisville, KY, United States, ${ }^{19}$ Faculty of Health Sciences, Department of Molecular Biology and Biochemistry, Simon Fraser University, Burnaby, BC, Canada, ${ }^{20}$ Department of Microbiology, Boston University School of Medicine, Boston, MA, United States, ${ }^{21}$ Department of Mathematics and Statistics, Boston University, Boston, MA, United States

High-throughput sequencing (HTS) of immunoglobulin (B-cell receptor, antibody) and T-cell receptor repertoires has increased dramatically since the technique was introduced in 2009 (1-3). This experimental approach explores the maturation of the adaptive immune system and its response to antigens, pathogens, and disease conditions in exquisite detail. It holds significant promise for diagnostic and therapy-guiding applications. New technology often spreads rapidly, sometimes more rapidly than the understanding of how to make the products of that technology reliable, reproducible, or usable by others. As complex technologies have developed, scientific communities have come together to adopt common standards, protocols, and policies for generating and sharing data sets, such as the MIAME protocols developed for microarray experiments. The Adaptive Immune Receptor Repertoire (AIRR) Community formed in 2015 to address similar issues for HTS data of immune repertoires. The purpose of this perspective is to provide an overview of the AIRR Community's founding principles and present the progress that the AIRR Community has made in developing standards of practice and data sharing protocols. Finally, and most important, we invite all interested parties to join this effort to facilitate sharing and use of these powerful data sets (join@airr-community.org).

Keywords: B-cell receptors, T-cell receptors, data sharing, immunogenetics, community standards, high-throughput sequencing, immunoglobulins, antibodies 


\section{INTRODUCTION}

The adaptive immune system provides protection against disease without inducing harmful autoimmunity; it reacts against the vast and ever-changing array of pathogens that an individual will encounter over a lifetime, while tolerating self. The variable regions of the adaptive immune receptors on $\mathrm{B}$ cells and $\mathrm{T}$ cells arise through the rearrangement of germline variable, diversity, and joining gene segments $(4,5)$. Humans each express over 100 million unique immunoglobulins (6) and a similar number of T-cell receptors $(1,7)$. The lymphocytes that express these receptors arise, proliferate, and die on time scales of hours to years $(1,8)$. Thus, the collection of B-cell and $\mathrm{T}$-cell receptor variable region genes expressed at any given time- - the adaptive immune receptor repertoire (AIRR) - is dynamic.

Immunoglobulin and T-cell receptor sequences have been studied for decades and several established databases exist including Kabat-Wu and Vbase2 $(9,10)$. Furthermore, there are databases that incorporate or allow viewing of structural data, such as IMGT, IEDB-3D, AntigenDB, and SAbDab [reviewed in Ref. (11)]. These data sets provide important insights into immune receptor-antigen interactions and can inform antibody engineering efforts. However, a single immunoglobulin or $\mathrm{T}$-cell receptor sequence is but a drop of water in the ocean that is the immune repertoire. While many immune repertoire studies have been performed using a variety of methods [reviewed in Ref. (12)], adequate analysis of the repertoire as a whole was virtually impossible prior to the advent of high-throughput sequencing (HTS). Here, we focus on HTS-based profiling of AIRR.

Since HTS was first applied to AIRR profiling in 2009 (1, 3, $6,7)$, there has been rapid advancement of both experimental and computational techniques. HTS of AIRRs (AIRR-seq) is yielding valuable insights into how variation in the AIRR differs across lymphocyte subsets (13-16) and anatomic compartments (17-20), varies over the course of a disease or with therapy (21-27), and is influenced by age (28-32), genetic background $(33,34)$, health status $(19,29,35-37)$, antigen exposure $(27,38-40)$, and other factors. AIRR-seq data are increasingly important in the development of vaccines, monoclonal antibodies, cancer immunotherapies, and other applications [reviewed in Ref. (41)]. As the number of datasets continues to grow, comparative analyses of hundreds or even thousands of individuals will soon be feasible. Ensuring the reliability of such integrative analyses, however, will require the establishment of and adherence to standards for reporting and sharing data across multiple laboratories and centers.

\section{CHALLENGES FOR AIRR-SEQ DATA SHARING}

Several challenges currently impede the effective sharing of AIRR-seq data. First, the storage and transport of such large datasets, which can comprise hundreds of millions of sequences (and hundreds of gigabytes) per study, require substantial time and resources. Second, deposition into public archives is not uniformly required by journals or funding agencies. As of
September 4, 2017, a Wiki page on the B-T.CR forum ${ }^{1}$ lists 82 AIRR-seq studies that report full HTS data to a public archive, ${ }^{2}$ while 42 (34\%) do not. ${ }^{3}$ Third, the information required to ensure appropriate use of such data by secondary users requires delineation (42). These challenges are not unique to AIRR-seq data. Indeed, the need for shared standards has been recognized and addressed for previous high-throughput technologies (43), including microarray data (44).

Another significant challenge for AIRR-seq data is that the processing pipeline between the experiment and the ultimate analysis of the data is lengthy and specialized (45-59). Beyond the steps required to process any HTS data, the annotation required of AIRR-seq data is unique to these genes and subject to substantial uncertainty (52). Unlike other genes, the antigen receptors of adaptive immunity are assembled through the recombination of randomly chosen gene segments, with nontemplated nucleotides added to the junctions and nucleotides nibbled away from the gene segments (60). In B cells, somatic hypermutation during affinity maturation results in further diversification of immunoglobulin genes $(61,62)$. In order for these data to be effectively shared and reanalyzed, the development of new metadata standards specific to the experimental and bioinformatic methods associated with AIRR-seq are required.

\section{A BRIEF HISTORY OF THE AIRR COMMUNITY}

The AIRR Community was established in 2015 at a meeting organized by Felix Breden, Jamie Scott, and Thomas Kepler in Vancouver, BC, USA to address these data sharing challenges. Membership in the AIRR Community is open and is intended to cover all aspects of AIRR-seq technology and its uses. Membership includes researchers expert in the generation of AIRR data; statisticians and bioinformaticians versed in their analysis; informaticians and data security experts experienced in their management; basic scientists and physicians who turn to such data for critical insights; and experts in the ethical, legal, and policy implications of sharing AIRR data.

In 2015, the AIRR Community formed three Working Groups. The Minimal Standards Working Group was tasked with the development of a set of metadata standards for the publication and sharing of AIRR-seq datasets. The Tools and Resources Working Group is focused on the development of standardized resources to facilitate the comparison of AIRR-seq datasets and analysis tools, including collection, validation, and nomenclature of germline alleles. Finally, the Common Repository Working Group is working to establish requirements for repositories that will store AIRR data. The Working Groups are dynamic and often collaborate with each other, as methods evolve and applications of standards in one area (for example, metadata standards) impact other areas (data repository requirements). Full recommendations and membership lists for the Working Groups as well as video recordings of the 2016 AIRR Community meeting are

\footnotetext{
${ }^{1}$ https://www.b-t.cr.

${ }^{2}$ https://www.b-t.cr/t/317.

${ }^{3}$ https://www.b-t.cr/t/426.
} 
available at http://www.airr-community.org. At the June 2016 meeting held at the National Institutes of Health (NIH), the AIRR Community ratified an initial set of recommendations that are summarized herein.

\section{DATA GENERATION}

Due to the complexity and diversity of the data sets being generated, the AIRR Community is developing best practices for the generation of AIRR-seq data. Such best practice guidelines will include, at a minimum: standard operating procedures for cell isolation and purification, including panels and gating strategies for flow cytometry; primers and protocols for amplification and sequencing of BCR or TCR rearrangements; and a clear description of library preparation and sequencing. Nomenclature is particularly important when it comes to the multiple stages of sample processing and data analysis. For example, what is meant by "raw data" differs among investigators, compounded by the fact that there are multiple levels of data preprocessing.

At present, the AIRR Community recommends that: (1) experimental protocols should be made available through a public repository granting digital object identifiers; (2) the change history of the experimental protocols, including details of what was changed and when the changes were made, should be made publicly available through the same repository; and (3) biological materials (e.g., plasmids, cell lines) should be made available to interested researchers via public repositories (e.g., Addgene for vectors, ATCC for cell lines), whenever possible.

\section{DATA SHARING}

For transparency and reliable reuse, experiments need to be sufficiently well annotated to allow evaluation of the quality of individual datasets and comparability of different datasets. Therefore, the AIRR Community has developed experimental metadata standards for AIRR-seq data generation, processing, and quality control. The data consist of the raw sequences and the processed sequences, while metadata include clinical and demographic data on study subjects and protocols for cell phenotyping, nucleic acid purification, AIRR amplicon production, HTS library preparation and sequencing, as well as documentation of the computational pipelines used to process the data. In publications or other forms of data sharing, these metadata sets and their components should be described in sufficient detail such that a person skilled in the art of AIRR sequencing and data analysis will be able to reproduce the experiment and data analyses that were performed. A manuscript describing a complete model for AIRR-seq data and metadata, and standardized terminology will soon be submitted.

Data sharing is also premised on the user's ability to locate and access the data. The AIRR Community recommends that all published AIRR-seq data be deposited in designated public repositories that adhere to the AIRR Community minimal standards guidelines, namely, that the data should be made available under the least restrictive terms possible. Limited exceptions to respect commercial interests in intellectual property rights are under consideration by the AIRR Community. To facilitate data sharing, the AIRR Community is also establishing an AIRR Data Commons, comprised of multiple, distributed repositories optimized for storing and querying AIRR-seq data, and supported by a centralized Gateway. Under such an intermediate distributed model (43), interoperability and effective data sharing are ensured because participating repositories will be required to comply with the community-established data and metadata standards and certain technical requirements.

\section{LEGAL AND ETHICAL CONSIDERATIONS}

Adaptive immune receptor repertoire-seq data can be subject to regulations regarding informed consent, intellectual property, and ethical treatment of research subjects. During the process of making AIRR-seq data publicly available, researchers typically would attest that they have sought appropriate informed consent or other authorization for sharing, where applicable. To reduce the potential for a breach of privacy of research subjects, medical and demographic metadata should to be structured in such a way that individual research subjects are not identifiable. Access to health information is regulated by national and international laws, such as the Health Insurance Portability and Accountability Act in the United States or the EU Regulation 2016/679 in the European Union, which requires medical information and personal identifiers to be safeguarded. For studies using AIRR-seq data from human subjects, data must be collected following a protocol that has been approved by the researcher's Institutional Review Board, which oversees human subjects' protections and ensures that all studies are performed in a legal and ethical manner (63). Human subjects must provide informed consent, and there should be broad agreement in the consent language regarding the confidentiality of medical information and the use of AIRR-seq data and metadata for future research. Without such provisions, the data in the database may be too constrained, with respect to time or breadth of investigation, to be usable by investigators other than the initial data generators.

Whenever data or other items of potential commercial value are shared with others, the individuals who generated and deposited the data should to be given proper credit. Hence, users of the database should, at a minimum, credit the data depositors in any publication or grant application. One mechanism whereby these rules could be followed is to create an online form that must be completed before access to the database is granted. Such a form would essentially be a contract for using the data. Enforcement of the terms of the contract could include monitoring of data use and denying access to the database should the terms of the datause agreement be violated.

To facilitate broad access to and use of AIRR-seq data, the data should be made available under the least-restrictive terms possible. The default data sharing policy should be to deposit data in a public domain database with no restrictions over deposit, access, storage, curation, or use. For data deposited in public domain databases/repositories, neither the depositors nor the repositories should be permitted to interfere with access to and use of the data by others, including through the assertion of any intellectual property rights. Exceptions to open data sharing may arise in circumstances in which open data sharing would come 
into conflict with the law, such as those pertaining to personal privacy and protected health information, or into conflict with decisions made by an Institutional Review Board.

\section{DATA ANALYSIS}

The AIRR Community strongly advocates the use of statistical methods for data analysis and hypothesis testing. Statistical methods systematically characterize error, quantify uncertainty, and provide a measure of confidence for inferences. Statistical methods also form the basis for data analysis in all other realms of biomedical and scientific research and should be adopted for AIRR-seq data. Expanded production of AIRR-seq data has been supported by a proliferation of computational tools for their processing and analysis, including tools for variable region gene annotation, inference of clonal history and partitioning and visualization (16, 46-52, 55, 56, 64-69). To encourage broad and well-informed use of these tools, the AIRR Community recommends that analysis software be released under an Open Source Initiative approved license, hosted on a publicly available website or repository with versioning, and be designed for modularity and inter-operability with other software. The AIRR Community will promote best practices in AIRR-seq data analysis by: (1) developing and publishing common criteria for the evaluation of statistical methods; (2) providing common "gold-standard" datasets of multiple types for use in software development, testing, and calibration; and (3) establishing best practices for data sharing and analysis software platforms.

\section{CONCLUSION}

Members of the AIRR Community have worked together for over 2 years with enthusiasm, driven by the belief that optimizing the reproducibility and sharing of AIRR-seq data will have a profound and positive effect on biomedical research and patient care. To encourage widespread adoption, the AIRR Community recommends that journals and funding agencies require AIRR-seq data be made available through a public data repository after publication or as negotiated in data-sharing agreements for unpublished

\section{REFERENCES}

1. Freeman JD, Warren RL, Webb JR, Nelson BH, Holt RA. Profiling the T-cell receptor beta-chain repertoire by massively parallel sequencing. Genome Res (2009) 19(10):1817-24. doi:10.1101/gr.092924.109

2. Robins HS, Campregher PV, Srivastava SK, Wacher A, Turtle CJ, Kahsai O, et al. Comprehensive assessment of T-cell receptor beta-chain diversity in alphabeta T cells. Blood (2009) 114(19):4099-107. doi:10.1182/blood-200904-217604

3. Weinstein JA, Jiang N, White RA III, Fisher DS, Quake SR. Highthroughput sequencing of the zebrafish antibody repertoire. Science (2009) 324(5928):807-10. doi:10.1126/science.1170020

4. Sakano H, Maki R, Kurosawa Y, Roeder W, Tonegawa S. Two types of somatic recombination are necessary for the generation of complete immunoglobulin heavy-chain genes. Nature (1980) 286(5774):676-83. doi:10.1038/ $286676 \mathrm{a} 0$

5. Sakano $\mathrm{H}$, Kurosawa $\mathrm{Y}$, Weigert $\mathrm{M}$, Tonegawa S. Identification and nucleotide sequence of a diversity DNA segment (D) of immunoglob- data. The success of this initiative is also critically dependent upon acceptance by the researchers who generate and use AIRR-seq data. While members of the AIRR Community have tried to be inclusive through developing contacts with researchers in the field and extensively advertising the annual meetings, there are likely to be many researchers who generate, analyze, and use AIRRseq data, who are not aware of the AIRR Community initiative. Community "buy in" results from creating data standards that are transparently developed through public discussion, robustly evaluated, and periodically updated as the field advances. This Perspective represents an open invitation to the larger scientific community to participate in and adopt the AIRR initiative. To that end, we welcome feedback on this Perspective and on the AIRR Community's efforts to date. Individuals interested in working on any facet of this important initiative are invited to attend, in person or online, the 2017 Community Meeting hosted by the NIH in Rockville, MD, USA, December 3-6, 2017. Most of all, we encourage anyone who is interested to join the AIRR Community ${ }^{4}$ and participate in the working groups.

\section{AUTHOR CONTRIBUTIONS}

FB, EP, JS, and TK conceived of and wrote the manuscript. All other authors contributed ideas and/or proposed revisions to the text. The AIRR Community Working Groups developed and wrote the recommendations described herein.

\section{ACKNOWLEDGMENTS}

Many of the ideas presented herein evolved over the course of AIRR Community meetings and Working Group meetings. The AIRR Community initiative and Community meetings were supported by CIHR, NIH (Jon Warren and Joe Breen), NIH R13-AI116349, P01-AI106697, R01-AI097403 and P30-CA016520, GenMab, The Antibody Society, CHAVI, the IRMACS Centre, Simon Fraser University, Illumina, Genentech, TTP Labtech, Grifols, and Amgen.

${ }^{4}$ join@airr-community.org.

ulin heavy-chain genes. Nature (1981) 290(5807):562-5. doi:10.1038/ 290562a0

6. Glanville J, Zhai W, Berka J, Telman D, Huerta G, Mehta GR, et al. Precise determination of the diversity of a combinatorial antibody library gives insight into the human immunoglobulin repertoire. Proc Natl Acad Sci U S A (2009) 106(48):20216-21. doi:10.1073/pnas.0909775106

7. Robins H. Immunosequencing: applications of immune repertoire deep sequencing. Curr Opin Immunol (2013) 25(5):646-52. doi:10.1016/j. coi.2013.09.017

8. McLean AR, Michie CA. In vivo estimates of division and death rates of human T lymphocytes. Proc Natl Acad Sci U S A (1995) 92(9):3707-11. doi:10.1073/pnas.92.9.3707

9. Wu TT, Kabat EA. An analysis of the sequences of the variable regions of Bence Jones proteins and myeloma light chains and their implications for antibody complementarity. J Exp Med (1970) 132(2):211-50. doi:10.1084/jem.132.2.211

10. Retter I, Althaus HH, Münch R, Müller W. VBASE2, an integrative V gene database. Nucleic Acids Res (2005) 33(Database issue):D671-4. doi:10.1093/ nar/gki088 
11. Dunbar J, Krawczyk K, Leem J, Baker T, Fuchs A, Georges G, et al. SAbDab: the structural antibody database. Nucleic Acids Res (2014) 42(Database issue):D1140-6. doi:10.1093/nar/gkt1043

12. Six A, Mariotti-Ferrandiz ME, Chaara W, Magadan S, Pham HP, Lefranc MP, et al. The past, present, and future of immune repertoire biology - the rise of next-generation repertoire analysis. Front Immunol (2013) 4:413. doi:10.3389/ fimmu.2013.00413

13. Mroczek ES, Ippolito GC, Rogosch T, Hoi KH, Hwangpo TA, Brand MG, et al. Differences in the composition of the human antibody repertoire by B cell subsets in the blood. Front Immunol (2014) 5:96. doi:10.3389/fimmu. 2014.00096

14. Wu YC, Kipling D, Leong HS, Martin V, Ademokun AA, Dunn-Walters DK. High-throughput immunoglobulin repertoire analysis distinguishes between human IgM memory and switched memory B-cell populations. Blood (2010) 116(7):1070-8. doi:10.1182/blood-2010-03-275859

15. Martin VG, Wu YB, Townsend CL, Lu GH, O’Hare JS, Mozeika A, et al. Transitional B cells in early human B cell development - time to revisit the paradigm? Front Immunol (2016) 7:546. doi:10.3389/fimmu.2016.00546

16. Bashford-Rogers RJ, Palser AL, Huntly BJ, Rance R, Vassiliou GS, Follows GA, et al. Network properties derived from deep sequencing of human B-cell receptor repertoires delineate B-cell populations. Genome Res (2013) 23(11):1874-84. doi:10.1101/gr.154815.113

17. Briney BS, Willis JR, Finn JA, McKinney BA, Crowe JE Jr. Tissue-specific expressed antibody variable gene repertoires. PLoS One (2014) 9(6):e100839. doi:10.1371/journal.pone.0100839

18. Meng W, Zhang B, Schwartz GW, Rosenfeld AM, Ren D, Thome JJC, et al. An atlas of B-cell clonal distribution in the human body. Nat Biotechnol (2017) 35(9):879-84. doi:10.1038/nbt.3942

19. Stern JN, Yaari G, Vander Heiden JA, Church G, Donahue WF, Hintzen RQ, et al. B cells populating the multiple sclerosis brain mature in the draining cervical lymph nodes. Sci Transl Med (2014) 6(248):248ra107. doi:10.1126/ scitranslmed.3008879

20. Sathaliyawala T, Kubota M, Yudanin N, Turner D, Camp P, Thome JJ, et al. Distribution and compartmentalization of human circulating and tissueresident memory T cell subsets. Immunity (2013) 38(1):187-97. doi:10.1016/j. immuni.2012.09.020

21. Heather JM, Best K, Oakes T, Gray ER, Roe JK, Thomas N, et al. Dynamic perturbations of the T-cell receptor repertoire in chronic HIV infection and following antiretroviral therapy. Front Immunol (2015) 6:644. doi:10.3389/ fimmu.2015.00644

22. Racanelli V, Brunetti C, De Re V, Caggiari L, De Zorzi M, Leone P, et al. Antibody $\mathrm{V}(\mathrm{h})$ repertoire differences between resolving and chronically evolving hepatitis C virus infections. PLoS One (2011) 6(9):e25606. doi:10.1371/journal.pone.0025606

23. Faham M, Zheng J, Moorhead M, Carlton VE, Stow P, Coustan-Smith E, et al. Deep-sequencing approach for minimal residual disease detection in acute lymphoblastic leukemia. Blood (2012) 120(26):5173-80. doi:10.1182/ blood-2012-07-444042

24. Weng WK, Armstrong R, Arai S, Desmarais C, Hoppe R, Kim YH. Minimal residual disease monitoring with high-throughput sequencing of $\mathrm{T}$ cell receptors in cutaneous T cell lymphoma. Sci Transl Med (2013) 5(214):214ra171. doi:10.1126/scitranslmed.3007420

25. Kalos M, Levine BL, Porter DL, Katz S, Grupp SA, Bagg A, et al. T cells with chimeric antigen receptors have potent antitumor effects and can establish memory in patients with advanced leukemia. Sci Transl Med (2011) 3(95):95ra73. doi:10.1126/scitranslmed.3002842

26. Morris H, DeWolf S, Robins H, Sprangers B, LoCascio SA, Shonts BA, et al. Tracking donor-reactive T cells: evidence for clonal deletion in tolerant kidney transplant patients. Sci Transl Med (2015) 7(272):272ra10. doi:10.1126/ scitranslmed. 3010760

27. Havenar-Daughton C, Carnathan DG, Torrents de la Peña A, Pauthner M, Briney B, Reiss SM, et al. Direct probing of germinal center responses reveals immunological features and bottlenecks for neutralizing antibody responses to HIV env trimer. Cell Rep (2016) 17(9):2195-209. doi:10.1016/j.celrep.2016.10.085

28. Russell Knode LM, Naradikian MS, Myles A, Scholz JL, Hao Y, Liu D, et al. Age-associated B cells express a diverse repertoire of VH and Vkappa genes with somatic hypermutation. J Immunol (2017) 198(5):1921-7. doi:10.4049/ jimmunol.1601106
29. Gibson KL, Wu YC, Barnett Y, Duggan O, Vaughan R, Kondeatis E, et al. B-cell diversity decreases in old age and is correlated with poor health status. Aging Cell (2009) 8(1):18-25. doi:10.1111/j.1474-9726.2008.00443.x

30. Qi Q, Liu Y, Cheng Y, Glanville J, Zhang D, Lee JY, et al. Diversity and clonal selection in the human T-cell repertoire. Proc Natl Acad Sci U S A (2014) 111(36):13139-44. doi:10.1073/pnas.1409155111

31. Rechavi E, Lev A, Lee YN, Simon AJ, Yinon Y, Lipitz S, et al. Timely and spatially regulated maturation of $\mathrm{B}$ and $\mathrm{T}$ cell repertoire during human fetal development. Sci Transl Med (2015) 7(276):276ra25. doi:10.1126/ scitranslmed.aaa0072

32. Guo C, Wang Q, Cao X, Yang Y, Liu X, An L, et al. High-throughput sequencing reveals immunological characteristics of the TRB-/IgH-CDR3 region of umbilical cord blood. J Pediatr (2016) 176:69-78.e1. doi:10.1016/j. jpeds.2016.05.078

33. Notarangelo LD, Kim MS, Walter JE, Lee YN. Human RAG mutations: biochemistry and clinical implications. Nat Rev Immunol (2016) 16(4):234-46. doi:10.1038/nri.2016.28

34. Watson CT, Steinberg KM, Huddleston J, Warren RL, Malig M, Schein J, et al. Complete haplotype sequence of the human immunoglobulin heavychain variable, diversity, and joining genes and characterization of allelic and copy-number variation. Am J Hum Genet (2013) 92(4):530-46. doi:10.1016/j. ajhg.2013.03.004

35. Tipton CM, Fucile CF, Darce J, Chida A, Ichikawa T, Gregoretti I, et al. Diversity, cellular origin and autoreactivity of antibody-secreting cell population expansions in acute systemic lupus erythematosus. Nat Immunol (2015) 16(7):755-65. doi:10.1038/ni.3175

36. Stamatopoulos K, Belessi C, Moreno C, Boudjograh M, Guida G, Smilevska T, et al. Over $20 \%$ of patients with chronic lymphocytic leukemia carry stereotyped receptors: pathogenetic implications and clinical correlations. Blood (2007) 109(1):259-70. doi:10.1182/blood-2006-03-012948

37. Rubelt F, Bolen CR, McGuire HM, Vander Heiden JA, Gadala-Maria $\mathrm{D}$, Levin $\mathrm{M}$, et al. Individual heritable differences result in unique cell lymphocyte receptor repertoires of naive and antigen-experienced cells. Nat Commun (2016) 7:11112. doi:10.1038/ncomms11112

38. LasersonU,VigneaultF, Gadala-MariaD, YaariG,Uduman M,VanderHeidenJA, et al. High-resolution antibody dynamics of vaccine-induced immune responses. Proc Natl Acad Sci U S A (2014) 111(13):4928-33. doi:10.1073/ pnas.1323862111

39. Vollmers C, Sit RV, Weinstein JA, Dekker CL, Quake SR. Genetic measurement of memory B-cell recall using antibody repertoire sequencing. Proc Natl Acad Sci U S A (2013) 110(33):13463-8. doi:10.1073/pnas.1312146110

40. Lavinder JJ, Wine Y, Giesecke C, Ippolito GC, Horton AP, Lungu OI, et al. Identification and characterization of the constituent human serum antibodies elicited by vaccination. Proc Natl Acad Sci U S A (2014) 111(6):2259-64. doi:10.1073/pnas.1317793111

41. Georgiou G, Ippolito GC, Beausang J, Busse CE, Wardemann H, Quake SR. The promise and challenge of high-throughput sequencing of the antibody repertoire. Nat Biotechnol (2014) 32(2):158-68. doi:10.1038/nbt.2782

42. Bhattacharya S, Andorf S, Gomes L, Dunn P, Schaefer H, Pontius J, et al. ImmPort: disseminating data to the public for the future of immunology. Immunol Res (2014) 58(2-3):234-9. doi:10.1007/s12026-014-8516-1

43. Contreras JL, Reichman JH. DATA ACCESS. Sharing by design: data and decentralized commons. Science (2015) 350(6266):1312-4. doi:10.1126/ science.aaa7485

44. Brazma A, Hingamp P, Quackenbush J, Sherlock G, Spellman P, Stoeckert C, et al. Minimum information about a microarray experiment (MIAME)-toward standards for microarray data. Nat Genet (2001) 29(4):365-71. doi:10.1038/ ng1201-365

45. Yaari G, Kleinstein SH. Practical guidelines for B-cell receptor repertoire sequencing analysis. Genome Med (2015) 7:121. doi:10.1186/ s13073-015-0243-2

46. Imkeller K, Arndt PF, Wardemann H, Busse CE. sciReptor: analysis of single-cell level immunoglobulin repertoires. BMC Bioinformatics (2016) 17:67. doi:10.1186/s12859-016-0920-1

47. Rosenfeld AM, Meng W, Luning Prak ET, Hershberg U. ImmuneDB: a system for the analysis and exploration of high-throughput adaptive immune receptor sequencing data. Bioinformatics (2017) 33(2):292-3. doi:10.1093/ bioinformatics/btw593 
48. Rogosch T, Kerzel S, Hoi KH, Zhang Z, Maier RF, Ippolito GC, et al. Immunoglobulin analysis tool: a novel tool for the analysis of human and mouse heavy and light chain transcripts. Front Immunol (2012) 3:176. doi:10.3389/fimmu.2012.00176

49. Ralph DK, Matsen FA IV. Likelihood-based inference of B cell clonal families. PLoS Comput Biol (2016) 12(10):e1005086. doi:10.1371/journal.pcbi.1005086

50. Bolotin DA, Poslavsky S, Mitrophanov I, Shugay M, Mamedov IZ, Putintseva EV, et al. MiXCR: software for comprehensive adaptive immunity profiling. Nat Methods (2015) 12(5):380-1. doi:10.1038/nmeth.3364

51. Shugay M, Bagaev DV, Turchaninova MA, Bolotin DA, Britanova OV, Putintseva EV, et al. VDJtools: unifying post-analysis of $\mathrm{T}$ cell receptor repertoires. PLoS Comput Biol (2015) 11(11):e1004503. doi:10.1371/journal. pcbi. 1004503

52. Kepler TB. Reconstructing a B-cell clonal lineage. I. Statistical inference of unobserved ancestors. F1000Res (2013) 2:103. doi:10.12688/f1000research.2-103.v1

53. Kepler TB, Munshaw S, Wiehe K, Zhang R, Yu JS, Woods CW, et al. Reconstructing a B-cell clonal lineage. II. Mutation, selection, and affinity maturation. Front Immunol (2014) 5:170. doi:10.3389/fimmu.2014.00170

54. Liberman G, Benichou JI, Maman Y, Glanville J, Alter I, Louzoun Y. Estimate of within population incremental selection through branch imbalance in lineage trees. Nucleic Acids Res (2016) 44(5):e46. doi:10.1093/nar/gkv1198

55. Vincent B, et al. iWAS - a novel approach to analyzing next generation sequence data for immunology. Cell Immunol (2016) 299:6-13. doi:10.1016/j. cellimm.2015.10.012

56. Volpe JM, Cowell LG, Kepler TB. SoDA: implementation of a 3D alignment algorithm for inference of antigen receptor recombinations. Bioinformatics (2006) 22(4):438-44. doi:10.1093/bioinformatics/btk004

57. Alamyar E, Duroux P, Lefranc MP, Giudicelli V. IMGT((R)) tools for the nucleotide analysis of immunoglobulin (IG) and $\mathrm{T}$ cell receptor (TR) V-(D)-J repertoires, polymorphisms, and IG mutations: IMGT/V-QUEST and IMGT/HighV-QUEST for NGS. Methods Mol Biol (2012) 882:569-604. doi:10.1007/978-1-61779-842-9_32

58. Ye J, Ma N, Madden TL, Ostell JM. IgBLAST: an immunoglobulin variable domain sequence analysis tool. Nucleic Acids Res (2013) 41(Web Server issue):W34-40. doi:10.1093/nar/gkt382

59. Zhang B, Meng W, Luning Prak ET, Hershberg U. Discrimination of germline $\mathrm{V}$ genes at different sequencing lengths and mutational burdens: a new tool for identifying and evaluating the reliability of $\mathrm{V}$ gene assignment. J Immunol Methods (2015) 427:105-16. doi:10.1016/j.jim.2015.10.009

60. Gellert M. V(D)J recombination: RAG proteins, repair factors, and regulation. Annu Rev Biochem (2002) 71:101-32. doi:10.1146/annurev.biochem.71. 090501.150203

61. Weigert MG, Cesari IM, Yonkovich SJ, Cohn M. Variability in the lambda light chain sequences of mouse antibody. Nature (1970) 228(5276):1045-7. doi: $10.1038 / 2281045 \mathrm{a} 0$
62. Jacob J, Kelsoe G, Rajewsky K, Weiss U. Intraclonal generation of antibody mutants in germinal centres. Nature (1991) 354(6352):389-92. doi: $10.1038 / 354389 \mathrm{a} 0$

63. Freedman RS, Cantor SB, Merriman KW, Edgerton ME. 2013 HIPAA changes provide opportunities and challenges for researchers: perspectives from a cancer center. Clin Cancer Res (2016) 22(3):533-9. doi:10.1158/1078-0432. CCR-15-2155

64. Gupta NT, Adams KD, Briggs AW, Timberlake SC, Vigneault F, Kleinstein SH. Hierarchical clustering can identify B cell clones with high confidence in ig repertoire sequencing data. JImmunol (2017) 198(6):2489-99. doi:10.4049/ jimmunol.1601850

65. Gupta NT, Vander Heiden JA, Uduman M, Gadala-Maria D, Yaari G, Kleinstein SH. Change-O: a toolkit for analyzing large-scale B cell immunoglobulin repertoire sequencing data. Bioinformatics (2015) 31(20):3356-8. doi:10.1093/bioinformatics/btv359

66. Vander Heiden JA, Yaari G, Uduman M, Stern JN, O’Connor KC, Hafler DA, et al. pRESTO: a toolkit for processing high-throughput sequencing raw reads of lymphocyte receptor repertoires. Bioinformatics (2014) 30(13):1930-2. doi:10.1093/bioinformatics/btu138

67. Ostmeyer J, Christley S, Rounds WH, Toby I, Greenberg BM, Monson NL, et al. Statistical classifiers for diagnosing disease from immune repertoires: a case study using multiple sclerosis. BMC Bioinformatics (2017) 18(1):401. doi:10.1186/s12859-017-1814-6

68. Toby IT, Levin MK, Salinas EA, Christley S, Bhattacharya S, Breden F, et al. VDJML: a file format with tools for capturing the results of inferring immune receptor rearrangements. BMC Bioinformatics (2016) 17(Suppl 13):333. doi:10.1186/s12859-016-1214-3

69. Christley S, Levin MK, Toby I, Fonner J, Monson N, Rounds WH, et al. VDJPipe: a pipelined tool for pre-processing immune repertoire sequencing data. BMC Bioinformatics (2017) 18(1):448. doi:10.1186/s12859-017$1853-\mathrm{z}$

Conflict of Interest Statement: The authors declare that the research was conducted in the absence of any commercial or financial relationships that could be construed as a potential conflict of interest.

Copyright (c) 2017 Breden, Luning Prak, Peters, Rubelt, Schramm, Busse, Vander Heiden, Christley, Bukhari, Thorogood, Matsen IV, Wine, Laserson, Klatzmann, Douek, Lefranc, Collins, Bubela, Kleinstein, Watson, Cowell, Scott and Kepler. This is an open-access article distributed under the terms of the Creative Commons Attribution License (CC BY). The use, distribution or reproduction in other forums is permitted, provided the original author(s) or licensor are credited and that the original publication in this journal is cited, in accordance with accepted academic practice. No use, distribution or reproduction is permitted which does not comply with these terms. 